\title{
Alter
}

Revue de phénoménologie

$18 \mid 2010$

L'Attention

\section{Expérimentations avec la réalité}

\section{Bernhard Waldenfels}

Traducteur : Flavien Le Bouter

\section{OpenEdition}

\section{Journals}

Édition électronique

URL : http://journals.openedition.org/alter/1748

DOI : $10.4000 /$ alter. 1748

ISSN : 2558-7927

Éditeur :

Association ALTER, Archives Husserl (CNRS-UMR 8547)

Édition imprimée

Date de publication : 1 octobre 2010

Pagination : 305-329

ISBN : 2-9522374-6-8

ISSN : 1249-8947

\section{Référence électronique}

Bernhard Waldenfels, «Expérimentations avec la réalité », Alter [En ligne], 18 | 2010, mis en ligne le 01 juin 2020, consulté le 29 juin 2020. URL : http://journals.openedition.org/alter/1748 ; DOI : https:// doi.org/10.4000/alter.1748 


\title{
EXPÉRIMENTATIONS AVEC LA RÉALITÉ*
}

\author{
Bernhard Waldenfels
}

\author{
"Je veux dire que dans la réalité se cache un désir insensé d'irréalité !" \\ Robert Musil, L'Homme sans qualités
}

Nous sommes depuis longtemps habitués à parler de réalités ou de mondes au pluriel. Au plan synchronique, le monde se divise en diverses réalités qui présentent chacune leurs propres types de sens, leurs propres règles, leurs propres conditions de constitution. Sur l'axe diachronique se rencontrent $\mathrm{d}^{\prime}$ anciennes et de nouvelles réalités qui s'entrelacent. À un horizon plus lointain se dessinent des mondes possibles avec lesquels nous jouons mais envers lesquels nous avons aussi des attentes. Le concept de réalité se dilue dans les processus de la réalisation, de la déréalisation et de la néoréalisation. Par là, la question de savoir comment le rapport à la réalité se modifie sous l'influence de médias omniprésents et de processus commandés par ordinateur perd sa dimension dramatique. En y regardant de plus près, " la réalité » qu'Ulrich, l'homme sans qualités, dans sa joie à expérimenter, aimerait abolir ${ }^{1}, \mathrm{n}^{\prime}$ a jamais existé même s'il a toujours semblé que le sens de la réalité éclipsait le sens de la possibilité. Pourtant, de cette façon, on ne répond pas à la question de savoir de quel type sont les possibilités qui pénètrent notre monde réel et quelle importance il faut leur accorder ; et cette question se pose avec une nouvelle urgence compte tenu de la multiplication des réalités artificielles.

Les réflexions qui suivent partent de la tension, exacerbée par la technologie, entre le sens de la réalité et le sens de la possibilité. La réalité, qui est toujours présupposée dans notre expérience et notre entente quotidiennes, se manifeste de façon expresse par les expérimentations avec la réalité. Ces

* L'article est paru dans le livre Medien, Computer, Realität. Wirklichkeitsvorstellungen und neue Medien, Sybille Krämer (éd.), Francfort/Main, Suhrkamp, p. 213-243. Nous remercions les éditions Suhrkamp pour leur aimable autorisation.

1. Musil, 1978, p. 289. 


\section{L'attention}

expérimentations portent indirectement atteinte au caractère de réalité en neutralisant certains présupposés de la normalité. Une série de telles anomalisations, qui sont à situer à la jonction du naturel et de l'artificiel, est envisagée de façon exemplaire en référence aux phénomènes de déviance induits pathologiquement et technologiquement. A l'horizon de ces réflexions se pose la question de savoir s'il n'y a pas quelques limites du pouvoir-faire, qui ne tiennent à la limitation des espaces de possibilités mais à des perturbations qui, sous la forme d'interventions ou d'exigences étrangères, constituent un contrepoids à leur propre pouvoir-faire ${ }^{2}$.

$\mathrm{Au}$ plan méthodologique, c'est par des voies indirectes que j'aborde les nouveaux médias et technologies qui participent aux expérimentations avec la réalité, en partant d'une phénoménologie de l'expérience vécue, intimement liée à une phénoménotechnique au sens de Gaston Bachelard ${ }^{3}$. Je vois le trait d'union entre une phénoménologie, qui rend compte de ce qui se montre, et une phénoménotechnique, qui met en évidence les conditions correspondantes, dans la jonction du quoi et du comment, des états de choses et $\mathrm{du}$ mode d'accès. Cette jonction exclut justement la possibilité d'accéder à la réalité et de compter sur une réalité donnée définitivement.

\section{Expérience de la réalité et langage de la réalité}

Si nous partons d'une expérience vécue, d'une modification, d'une invention, d'une constitution ou d'une construction de la réalité ou si nous évoquons une perte de la réalité, se pose d'abord la question de savoir de quel type de réalité il est question. En y regardant de près, il apparaît que nous ne pouvons parler de réalité que de manière indirecte, en parlant en même temps de beaucoup d'autres choses.

\section{La réalité comme modalité impliquée}

La réalité apparaît tout particulièrement aux deux extrémités de notre vécu et de notre comportement, dans la perception charnelle dans laquelle quelque chose nous est donné comme réel et dans l'action charnelle dans laquelle nous produisons quelque chose. Étant donné que remarquer (bemerken) et produire (bewirken) s'imbriquent de façon circulaire, nous ne devons pas poser deux réalités, l'une cognitive et l'autre pratique, mais nous devons partir d'une réalité unique qui est à la fois donnée et engendrée. La réalité (Wirklichkeit) est liée à l'efficacité (Wirksamkeit). Sur ce point, de nombreuses théories s'accordent même si elles déterminent ou évaluent diversement les facteurs de cette indissociabilité. On peut néanmoins se

2. Je renvoie à ce sujet à la version détaillée de cet article qui est parue dans mon livre Grenzen der Normalisierung. Studien zur Phänomenologie des Fremden 2, comme à l'horizon problématique que j'ai déterminé dans Antwortregister.

3. Des informations plus précises au sujet de Bachelard se trouvent in Waldenfels 1983, p. 381. 
demander ce que signifie la réalité. Au plan linguistique, comme la "mortalité », la "réalité » semble indiquer une qualité. Comme l'energeia grecque, l'expression " effectivité (Wirklichkeit)» évoquerait l'agir-sur (wirken) alors que la " réalité (Realität) » (du latin realitas) signifie littéralement ce qui constitue une res et, pour parler généralement, ce qui fait qu'une « chose » ou un « quelque chose » est ce qu'il est. Pourtant, des analyses sémantiques, intentionnelles ou pragmatiques montrent de manière concordante que la prudence est ici de mise. «Effectif» et «réel» ne sont ni de véritables prédicats trouvant leur application dans des énoncés singuliers d'existence ni des données originelles qu'on peut mettre en évidence dans l'expérience vécue ni un résultat d'action ou un produit fabriqué que nous posons nousmêmes dans le monde.

Selon Kant, le concept de réalité n'ajoute rien aux cent thalers possibles. Dans des énoncés d'existence, comme «Homère, l'auteur de l'Iliade a existé », «J'existe», «Tu existes » ou «C'est réel », je suppose déjà l'existence réelle que je semble affirmer. Une négation de ces prétendues affirmations ne serait pas fausse mais dénuée de sens ou insignifiante ${ }^{4}$. Nous attribuerions l'épopée transmise à un auteur qui n'a jamais existé ou nous montrerions quelque chose qui n'existe pas du tout. L'analyse intentionnelle de la perception va dans le même sens. Je ne perçois pas quelque chose de réel comme je pourrais voir ou entendre également quelque chose d'irréel ; mais quelque chose de perçu présente en tant que tel le caractère ontologique de « réalité ${ }^{~}$ ». Qui croit percevoir, croit eo ipso à la réalité du perçu; si la croyance devait se révéler illusoire, on n'aurait pas perçu quelque chose d'irréel ; on aurait plutôt rien perçu du tout ou on aurait perçu quelque chose d'autre que ce qu'on croyait percevoir. Merleau-Ponty dit en ce sens : «Il ne faut pas se demander si nous percevons vraiment un monde, il faut dire au contraire : le monde est cela que nous percevons ${ }^{6}$. » $C^{\prime}$ est la même chose pour l'action et la production; elles ne créent pas quelque chose de réel, mais elles sont elles-mêmes une forme de la réalisation (Verwirklichung). Le pragmaticien William James ${ }^{7}$ rejoint la philosophie analytique et les

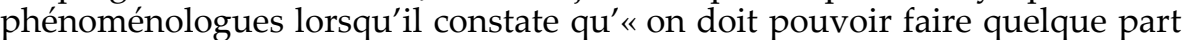
l'expérience de tout ce qui est réel et tout type de chose dont on fait l'expérience doit être quelque part réel ». Même un constructiviste radical comme Ernst von Glasersfeld, qui se rapporte à James ${ }^{8}$ sur ce point fait une différence entre "faire l'expérience", qui retient son attention, et une

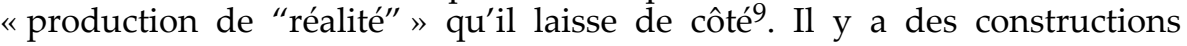

4. Voir le débat déclenché par B. Russell et P. Strawson au sujet de la référence des énoncés singuliers d'existence et leur résumé in Tugendhat, 1976, 22. Vorlesung ou Tugendhat et Wolf, 1993, chap. 11.

5. Hua, tome III, § 103 .

6. Merleau-Ponty, 1945, p. XI.

7. James, 1976, p. 81.

8. Ibid., p. 12.

9. Glasersfeld, 1996, p. 84. 


\section{L'attention}

réussies et d'autres qui ne le sont pas ; certaines qui sont viables et d'autres qui ne le sont pas ; c'est tout. C'est surtout au bureau administratif du pays de Cocagne que se trouvent des usines à réalité ${ }^{10}$.

La réalité ne peut donc être conçue comme quelque chose ou comme la caractéristique de quelque chose mais doit être comprise comme modalité, c'est-à-dire comme mode d'être, mode de donné, comme mode linguistique de relation ou comme mode de production d'un type particulier. À cela s'ajoute le fait que le caractère de réalité ou le caractère de croyance correspondant ne représente pas un mode d'être parmi d'autres, mais est impliqué dans toutes les modalisations et toutes les modifications en tant que «mode originaire ${ }^{11}$ ». Le caractère implicationnel de la réalité a pour conséquence que les expérimentations qui sont réalisées avec l'expérience charnelle (leiblich) porte eo ipso atteinte au caractère de réalité des choses. Le présent en chair et en os (leibhaftig) des choses et la dimension charnelle de notre soi s'interpénètrent dans l'expérience, et cela vaut aussi pour leurs perturbations réciproques.

\section{La foi en la réalité}

En accord avec David Hume, Husserl parle d'une foi perceptive qui, en tant que «doxa originaire (Urdoxa)», ne précède pas seulement toute tentative de fondation mais aussi toute prise de position, toute affirmation, toute négation, toute interrogation, toute mise en doute ${ }^{12}$. Le primat de la théorie de la connaissance est par là battu en brèche. Ce qui est réel est ce dont nous partons même si, dans le détail, nous le mettons en doute. Cette foi en la réalité est liée à la familiarité et à la fiabilité qui précèdent toute tentative de s'assurer (Vergewisserung). Les prédonnées de l'expérience perdent de leur importance si les constructions foisonnent. Pourtant, même le constructiviste Ernst von Glasersfeld, qui renonce à tout «savoir de la réalité ${ }^{3}$ », convient que la réalité peut se faire remarquer dans $\mathrm{l}^{\prime}$ empêchement ${ }^{14}$. Dans ce cas, est réel ce contre quoi nos constructions s'affirment ou ce sur quoi elles échouent. En tous les cas, la réalité ne serait nullement ce à quoi nous pouvons nous rapporter de manière séparée.

10. La distinction qu'on rencontre chez les constructivistes entre une « réalité » indépendante et une « réalité » construite ravive cependant les vieux problèmes d'un réalisme gnoséologique. À ce sujet, voir les réflexions critiques d'Arno Ros envers le constructivisme radical (in Rusch et Schmidt, 1994, p. 180-188).

11. Voir Hua, tome III, § 104 .

12. Hua, tome III, $\S 103$.

13. Glasersfeld, 1996, p. 315.

14. Ibid., p. 129 sq., p. 193. 


\section{L'expérience en relation}

Selon Husserl, tout étant qui vient à notre rencontre dans l'expérience est inscrit dans des horizons d'expérience spatio-temporels déterminés qui ont leur équivalent dans des situations d'action et des contextes de discours. Je n'entends pas des sons séparés mais des mélodies, des accords ou des bruits qui ressortissent vis-à-vis de l'arrière-plan du silence. De même, la simple ouverture d'une fenêtre ne se réduit pas à une quantité dénombrable de mouvements corporels séparés; cette tâche s'accomplit au contraire sous la forme d'une configuration de mouvements qui passe par certaines phases, qui présente un certain rythme et s'achève par un résultat d'ensemble, à savoir l'ouverture de la fenêtre. Lorsque nous percevons quelque chose, ce n'est pas seulement en plus que nous percevons quelque chose d'autre, comme le présuppose une théorie empiriste de la mosaïque ; nous percevons au contraire quelque chose en relation 15 . Quelque chose est donc ce qu'il est en se déterminant et en se conservant dans des relations changeantes ${ }^{16}$. La réalité est donc toujours en jeu dans l'expérience. Est réel ce à quoi je peux « toujours revenir ${ }^{17}$ ». Une expérience ponctuelle sans répétitions serait une expérience choc qui ferait un trou dans le tissu de l'expérience mais qui ne serait pas elle-même capable d'une détermination et d'une mise à l'épreuve. Si quelque chose est arraché à ses relations, cela implique une déréalisation partielle. Les relations font référence à d'autres relations sous la forme d'un infini ouvert. «Le réel s'offre à une exploration infinie ; il est inépuisable ${ }^{18} »$. Husserl désigne par monde le complexe global de renvois de notre expérience. La foi perceptive se présente en conséquence comme une foi dans le monde. Au monde n'appartient pas seulement ce dont on fait actuellement l'expérience mais aussi ce dont on peut potentiellement faire l'expérience et qui est esquissé plus ou moins dans l'expérience. À partir de cette possibilité de faire l'expérience de (Erfahrbarkeit) se distinguent des possibilités réelles de possibilités simplement logiques, pensées ${ }^{19}$. Au lieu de monde, on parle habituellement aussi de réalité ou d'une réalité dans sa totalité. Il faut pourtant ici être prudent. Si l'on attribue au complexe de renvois doué de sens qu'est le monde la représentation d'une omnitudo realitis, c'est-à-dire une somme de toutes les choses réelles, on répète à grande échelle la vieille erreur d'employer « réel » comme prédicat.

15. Au niveau neurologique, à cela correspond la formation de zones cérébrales secondaires qui sont responsables de l'identification des formes optiques et acoustiques et de la formation de modèles complexes. Voir Lurija, 1992, p. 109 sq, 129 sq.

16. Voir Hua, tome I, § 26.

17. Ibid., § 27.

18. Merleau-Ponty, 1945, p. 374.

19. Hua, tome III, $\S 47 \mathrm{sq}$. 


\section{L'attention}

\section{Le relief de l'expérience}

Dire que quelque chose est plus réel qu'autre chose a-t-il un sens ? La réalité est-elle susceptible d'accroissement? Ce n'est pas certain si seul est réel ce qui est le cas. Pour les états de fait qui sont enregistrés ou provoqués, l'un vaut autant que l'autre. Un meurtre n'est pas plus réel qu'une gifle; et un bulletin météorologique n'a pas moins de réalité que l'annonce de catastrophes. Cela vaut tant que les choses ne nous concernent pas du tout ou nous sont indifférentes. Les choses changent si des intérêts, des besoins ou des émotions entrent en jeu et si les choses sont d'importance plus ou moins grande, si elles nous interpellent ou nous concernent plus ou moins fortement et nous sont ainsi plus ou moins proches. L'expérience présente un relief dans lequel une chose fait saillie et l'autre se retire de telle sorte que nous sentons appelés à répondre. Cette saillie et ce retrait, par exemple de formes chromatiques, qui a son support physiologique dans le tonus musculaire ${ }^{20}$ et dont le pilotage neurologique se situe dans le tonus cortical $^{21}$, appartient aux formes de réalisation (Verwirklichungsformen) de l'expérience et non aux simples états affectifs qui accompagnent les cognitions et les actions. Dans la forme de l'attention qui privilégie en permanence certaines données et en défavorisent $\mathrm{d}^{\prime}$ autres ${ }^{22}$, ces privilèges pénètrent jusqu'au noyau le plus intime de la perception, de la pensée et de l'agir. Comme le disait déjà William James ${ }^{23}$ : "Chaque monde possède, lorsqu' on se tourne vers lui, son propre type de réalité; seul sa teneur en réalité diminue avec la diminution de l'attention. » Alfred Schütz, qui se réfère à James et à l'attention à la vie de Bergson, développe à partir de là sa théorie des réalités multiples au sein desquelles le monde quotidien, en tant que monde de l'agir-sur (Wirken), conserve le caractère d'une "réalité insigne ». Les prétentions à la réalité et leur importance peuvent être prises en considération de différentes manières. Il n'en reste pas moins qu'une expérience sans relief ne serait plus une expérience puisqu'elle sombrerait dans la monotonie. Faire l'expérience de la même manière, ce n'est faire l'expérience de rien. D'un point de vue fonctionnel, cette monotonie équivaut à un système homéostatique qui se trouve en équilibre. Toutefois, cette monotonie vécue se caractérise par le fait que la personne concernée souffre d'un sentiment d'ennui, ainsi que le dit le Danton de Büchner : "C'est très ennuyeux d'enfiler toujours la chemise d'abord puis la culotte par-dessus, et le soir de se glisser au lit et le matin de s'en extirper à nouveau... »

20. Goldstein, 1934, p. 167-174.

21. Lurija, 1992, p. 40 sq.

22. Cf. Hua, tome III, § 92 .

23. James, 1950, tome II, p. 293. 


\section{Les filtres de normalisation}

L'extension et l'intensification de l'expérience s'accroissent avec leur normalisation. Tout ce qui vient à notre rencontre dans l'expérience n'a pas la même valeur de réalité; n'est réel que ce qui correspond à certains standards. L'expérience se scinde en orthoesthésie et en hétéroesthésie, l'agir en orthopraxie et en hétéropraxie et le logos universel se divise en orthologie et en hétérologie ${ }^{24}$. La normalité et les anomalies se scindent si des désaccords se produisent dans l'expérience. Ces désaccords peuvent prendre différentes formes que nous connaissons déjà en partie depuis la thèse de l'homo mensura de Protagoras. Ainsi, la structure de la perception se modifie-t-elle selon les circonstances objectives, par exemple selon la manière dont elle créée par le biais de l'éclairage ou du bruitage. Elle se modifie lorsqu'un medium étranger, par exemple des lunettes coloriées, est intercalé25. Enfin, elle se modifie selon les circonstances charnelles-corporelles qui, dans le cas de strabisme, de cécité ou de prise de drogues, changent les phénomènes perceptifs. Husserl nomme « conditionnalité psychophysique » les circonstances charnelles dans lesquelles la subjectivité et la réalité neurophysiologique sont entrelacées. Dans ce domaine, il s'agit de désaccords intramodaux (vision en double ou blessure d'une main), de discordances intermodales (comme par exemple entre le sens sain du toucher et le sens altéré de la vue); il existe enfin des divergences intercharnelles (des manières de voir ou des formes hétérogènes de mouvement) qui se répercutent jusque dans le domaine culturel.

Selon Husserl, ces discordances restent limitées puisqu'il présuppose un «état originaire ${ }^{26}$ » de conditionnalités psychophysiques et conçoit la constitution normale comme la «première réalité du monde et de la chair ${ }^{27}$ » à laquelle renvoient toutes les anomalies. Les discordances sont en outre limitées puisque, selon Husserl, les divergences qui surviennent tiennent, d'une part, à la différence entre la normalité et les déviances pathologiques et, d'autre part, à un optimum pragmatique ; il compte ainsi sur la fonction unificatrice d'une idée régulatrice. De telles échappatoires ne peuvent pas d'emblée convaincre. Pour Husserl, « une même chose ne peut avoir des qualités discordantes ${ }^{28} »$. Qui argumente de la sorte identifie la réalité à la cohérence d'une expérience globale possible qui soigne toutes les fractures

24. Sur l'orthoesthésie et l'hétéroesthésie, voir les réflexions de Husserl au sujet de la constitution de la chose (Hua, Bd. IV, § 18) ainsi qu'au sujet de la normalité solipsiste et intersubjective (Hua, Bd. XIII, texte $\left.\mathrm{n}^{\circ} 14\right)$. Sur la préférence accordée à certains modes sensorimoteurs de comportement qui, en tant que "comportement insigne ", dépassent ce qui est physiologiquement réalisable, on trouve de nombreux exemples chez Goldstein, 1934, p. 219-239.

25. Sur le rôle des médias physiques dans l'expérience voir Hua, tome IV, p. 53 sq.

26. Hua, tome IV, p. 66.

27. Ibid., p. 68

28. Hua, tome XIII, p. 380. 


\section{L'attention}

de l'expérience. Plus important que cette pensée unificatrice douteuse est cependant le fait que, avec ces réflexions, des facteurs d'ordre pénètrent jusque dans le domaine de l'expérience sensori-charnelle et qu'ils constituent un «logos du monde esthétique ${ }^{29}$ ». Ce dont nous faisons l'expérience comme réel n'est jamais exempt de processus de normalisation; en ce sens, on n'en finit jamais $\mathrm{d}^{\prime}$ " arranger » la réalité. Avec la transformation des ordres de l'expérience, c'est aussi la réalité qui se transforme. Elle peut devenir obsolète, comme dans le cas de Don Quichotte auquel Alfred Schütz a consacré un essai remarquable ; mais elle peut aussi être prématurée. Le contraste entre un ordre ancien et un nouvel ordre mène d'une part à la fixation de la réalité et d'autre part à la dissolution de la réalité. Des déréalisations peuvent être compensées par des surréalisations qui compensent le manque de réalité. Par là, nous aboutissons au domaine des expérimentations qui touchent le nerf de la réalité.

\section{Les expérimentations de la réalité}

Les expérimentations ont affaire aux expériences, mais de telle sorte que les connaissances issues de l'expérience sont acquises par des interventions dans le monde de l'expérience. C'est ainsi qu'on peut lire dans le Novum Organum $^{30}$ de Francis Bacon que "restat experientia mera, quae, si occurat, casus, si quaesita sit, experimentum nominatur». La dualité ici nommée correspond au double sens de «faire des expériences »; on peut éprouver des expériences ou les provoquer. Les deux variantes de l'expérience ne pourraient alors être séparées l'une de l'autre soigneusement que si nous étions maîtres de notre expérience. Pourtant, ce ne sont pas seulement les appareils et les médias, poussant au-dessus de nos têtes et nous glissant entre les mains, qui imposent des limites à cette maîtrise. Des idées qui nous viennent à l'esprit, qui contrarient nos intentions et pénètrent dans le domaine familier, s'accompagnent déjà du fait que ce qu'on trouve, ce qui advient (in-venire), agresse, excède et dupe toute quête. Les idées qui nous viennent à l'esprit (Einfälle) ont quelque chose à voir avec les hasards (Zufälle) et les agressions (Überfälle); elles ne sont jamais anodines dès lors qu'elles vont au-delà des bêtises et des chimères. Nous pouvons donc distinguer des expérimentations survenant spontanément et des expérimentations provoquées artificiellement. Il importe ici de ne pas perdre de vue leur rapport mutuel. Kurt Goldstein ${ }^{31}$ indique qu'il existe une certaine identité entre les phénomènes produits expérimentalement et les phénomènes pathologiques dans la mesure où, dans les deux cas, l'isolement, par exemple la dissociation des stimuli et des réactions du comportement global, joue un rôle. En conséquence, au sein de ce qu'on exécute artificiellement, le hasard

29. Hua, tome XVII, p. 297.

30. I, 82.

31. Goldstein, 1934, p. 106. 
rattrape finalement toujours l'expérimentation. Nous ne serions maîtres de la situation que si nous pouvions métamorphoser le monde en un laboratoire clos. Cela serait l'équivalent technologique d'une métamorphose de la doxa en une épistémè scientifique qui, selon Husserl, est le propre de la crise des sciences européennes.

Par expérimentations de la réalité, nous désignons alors les expérimentations qui ne mettent pas quelque chose à l'épreuve dans l'expérience, mais qui soumettent la structure propre de l'expérience à une mise à l'épreuve. Si nos réflexions préliminaires sont justes et si nous pouvons à bon droit présupposer que le caractère de réalité n'est saisissable qu'indirectement, en tant que modalité impliquée de notre expérience, une expérimentation avec la réalité ne peut elle aussi avoir lieu que sous forme indirecte. De telles expérimentations ont pour point de départ les conditions normalisatrices de l'expérience qui établissent ce qui vaut comme réel. Elles impriment à l'expérience certains ordres dans lesquels la foi perceptive s'articule et dans lesquels des relations d'expérience sont sélectionnées et des critères de pertinence sont établis. La dé-réalisation ne signifie jamais simplement que quelque chose disparaît ; cela équivaut à un désordonnancement (Entordnung) de la même manière qu'une re-réalisation équivaut à un nouvel ordonnancement ${ }^{32}$ (Neuordnung). Les expérimentations avec la réalité peuvent donc être décrites comme des processus de l'anomalisation dans lesquels notre appréhension de la réalité se modifie. Puisque tout ce qui nous rencontre est toujours saisi comme quelque chose, c'est par là la réalité ellemême qui se modifie elle aussi indirectement.

Les expérimentations avec la réalité peuvent prendre pour point de départ des domaines d'ordre et des sphères de sens les plus divers. À cela appartiennent tout $\mathrm{d}$ 'abord les multiples formes de la pathologie dans lesquelles les déviances par rapport au normal sont vécues et traitées comme de la souffrance. Les déviances pathologiques, y compris les dommages cérébraux pathologiques, jettent une lumière particulière sur les formes normales du vécu et du comportement ainsi que sur les effets pathogènes issus d'une normalisation excessive. Il faut en outre ici mentionner les expérimentations artistiques. La libération de la couleur dans l'impressionnisme et le pointillisme se heurte aux conventions d'une conception selon laquelle les couleurs sont traitées comme une couleur locale et les éclairages changeants comme des phénomènes accessoires. Le Monsieur Teste de Valéry franchit déjà le seuil de ces expériences de pensée qui jouent un rôle central de la caverne de Platon jusqu'à Husserl et Wittgenstein en passant par le doute de Descartes et l'hypothèse d'un état de nature chez Hobbes. Prenons l'exemple de Husserl. L'épochè phénoménologique, qui vise à neutraliser les évidences, et la réduction eidétique, qui se sert du procédé d'une variation de l'imagination, peuvent être décrites comme des anomalisations ciblées. Pour Husserl, la réflexion phénoménologique a au

32. Cf. Waldenfels, 1985, chap. 12. 


\section{L'attention}

début quelque chose d'un «habitus contre-nature 33 ». Des expérimentations fondatrices se produisent partout où intervient de l'extra-ordinaire qui échappe aux différents ordres. Les expérimentations technologiques constituent un dernier champ d'expérimentation. Indépendamment du fait que toute expérimentation possède une note technique, les expérimentations technologiques déterminent dans une certaine mesure notre présent. Des formules comme "construction », " découverte » ou " mise en scène » de la réalité ont pris depuis longtemps une tonalité interdisciplinaire sous l'influence d'une technique et d'une technologie généralisées.

Dans ce qui suit, nous présenterons quelques expérimentations qui, à la jonction de la phénoménologie, de la pathologie et de la technologie mettent en évidence des points névralgiques de l'expérience. L'intégration des phénomènes pathologiques n'a rien à voir avec une tendance à la généralisation de la pathologie, mais elle résulte de faits déjà mentionnés, à savoir du fait que des situations extrêmes ou exceptionnelles, provoquées artificiellement, comportent des similitudes structurelles avec des maladies et des troubles naturels qui apparaissent habituellement spontanément ${ }^{34}$. En outre, les frontières entre la science-fiction et la technoscience réelle sont perméables lorsque cette dernière entraîne une extension infinie et sans but $\mathrm{du}$ domaine technique. La technique apparaît alors comme la réalisation des fictions scientifiques. Le vol d'Icare pourrait être considéré rétrospectivement comme une simulation rudimentaire, habillée de mythologie. Par là disparaîtrait aussi bien sûr l'effet d'avertissement qui revient dans $L a$ Chute d'Icare de Breughel sous des auspices modernes. Les utilisateurs du programme de réalité virtuelle «Icare» qui permet des vols corporels audessus d'un paysage virtuel, ont tout au plus à craindre que le programme ne se plante.

\section{Les effets d'anomalisation}

Les effets d'anomalisation, qui se joignent à la réalité et à la dimension charnelle de notre expérience, apparaissent comme des effets en chaîne et produisent de multiples façons des effets inverses ${ }^{35}$. Si la réalité n'est pas quelque chose qui est d'emblée fixée, de tels effets ne peuvent manquer de se révéler ambivalents. L'étrangeté de nouvelles expériences a pour prix des désarrois et des pertes. Le renvoi à la normalité apaisante d'hier n'est qu'une illusion d'optique. On oublie facilement que ce qui a fait ses preuves a connu

33. Hua, tome XIX/I, p. 16.

34. « Naturel» est ici à comprendre au sens de la «langue naturelle». En tant que découverte culturelle, la langue est tout à fait artificielle; elle se distingue pourtant de la «langue artificielle » introduite méthodologiquement.

35. À ce sujet, voir les effets antagonistes, par exemple les inversions réflexes dans les centrages insuffisants qui sont constatés dans le domaine neuronal (Goldstein, 1934, p. 174-187). 
un devenir et l'on est enclin à retoucher les franges et les fractures qui ont été ouvertes.

\section{Les perturbations de contexte}

Lorsqu'on parle de relations, d'horizons ou de contextes de l'expérience, nous voulons dire que quelque chose apparaît ensemble avec autre chose. La réalité que nous attribuons à ce dont nous faisons actuellement l'expérience, doit être comprise comme une coréalité. Si on porte atteinte à celle-ci, cela affecte la réalité elle-même. Les formations de contexte sélectionnent d'après leur être puisque les structures perceptives, les expressions propositionnelles et les conséquences de l'action, en dehors du cas des situations restreintes artificiellement telles qu'elles apparaissent dans les recherches en laboratoire, offrent plus de possibilités de connexion que celles qui sont réalisées. Cela mis à part, les contextes sont plus ou moins riches selon le nombre de rapports de sens envisagé. Le rétrécissement des contextes peut aboutir dans des cas extrêmes à un isolement des données et des processus partiels, à une simple "relation de juxtaposition (Und-Zusammenhang) » de telle sorte que l'un n'est plus relié intimement à l'autre et qu'il atteint un état d'absence et d'incapacité de connexion. Par là, nous nous rapprochons d'expériences choc ponctuelles, dans lesquelles, au sens strict, aucune expérience n'a lieu puisqu'on ne fait littéralement l'expérience de rien, c'està-dire pas l'expérience de quelque chose. Faire l'expérience de quelque chose présuppose en effet qu'il se distingue d'autre chose et, qu'en tant que tel, qu'il se répète dans des relations qui alternent. D'un point de vue interne, nous tombons dans le domaine d'une expérience mystique ineffable qui sombre dans le silence. D'un point de vue externe, à cela correspond ce que Heinz von Foerster appelle le «principe de la codification indifférenciée »; l'intensité de la production d'excitation ne dit rien de la qualité de l'excitation et encore moins de la constitution des choses. La réalité se délite en un $X$ indéterminé qui devrait en ce sens être tenu pour irrationnel ${ }^{36}$. L'isolement est l'opposé extrême d'une totalisation. La surcharge des contextes confine à un contexte global, c'est-à-dire à un tout au sein duquel tout est relié à tout. Le monde se fige en une image close du monde ${ }^{37}$.

36. Cf. Foerster, 1993, p. 31, Glasersfeld, 1996, p. 190. Avec l'hypothèse d'un X inconnaissable, le physicalisme se retourne en mysticisme, comme on le voit chez Theodor Fechner et Ernst Mach. Mais également pour un néokantien comme Heinrich Rickert (1913, p. 322), la réalité signifie quelque chose $\mathrm{d}^{\prime}$ « irrationnel » dans la mesure où elle se soustrait à tout concept.

37. La bipolarité de la perturbation de contexte trouve un soutien dans le domaine neurologique. Dans la tentative d'exprimer neurobiogiquement les lois de la psychologie de la forme, Gerald M. Edelman et ses collaborateurs emploient le concept de «complexité neuronale ». «La complexité est nulle lorsque tous les neurones sont reliés les uns aux autres, c'est-à-dire dans le cas d'une complète intégration. Elle est également nulle lorsqu'il n'existe aucune liaison entre les neurones, c'est-à-dire dans une complète ségrégation. Dans le domaine 


\section{L'attention}

Un façonnage artificiel des contextes peut s'adjoindre dans le pilotage de la sélection; et il est d'autant plus efficace que le processus de sélection reste dans l'obscurité et que rien ne nous aide à le voir. Husserl indique qu'une chose qui est extraite de sa relation aux choses ne nous offre plus aucun moyen de distinguer la chose du simple fantôme, c'est-à-dire d'une simple configuration spatiale comme l'arc-en-ciel ou le mirage ${ }^{38}$. Quelque chose de similaire nous arrive dans les relations techniques. Nous sommes livrés dans une certaine mesure à une suite d'images qui sont produites par les appareils et que nous déclenchons par une pression sur un bouton. Une vieille proposition dit qu'à l'inverse du ouï-dire, dans lequel nous faisons confiance à des mots et à des sens étrangers, la vision s'effectue avec ses propres yeux, sous la forme d'une autopsie ${ }^{39}$ qui est soutenue par des contacts palpables. Cette autopsie fait place à une hétéropsie, à une vision avec ses yeux étrangers, lorsque nous regardons une émission en direct ou que nous sommes en interaction avec elle. Dans cas, nous avons affaire à un présent charnel (leibhaft) à distance, à une téléprésence. Il existe bien sûr, quand " on y regarde à deux fois", des critères de crédibilité semblables à ceux de ce qu'on trouve dans l'audition. Pourtant, si nous ne participons pas nous-mêmes à la production des images, ces critères ont un caractère indirect, à la différence du cas des mirages qui se dissolvent devant nos yeux dans le néant. Comme dans le cas des trucages d'images, il s'agit ici de trucages de la perception. Un trucage de la perception est quelque chose d'autre qu'une illusion perceptive qui peut elle être percée à jour en tant que telle. Mais elle est aussi quelque chose d'autre qu'une erreur de la perception qui se corrige au fil de l'expérience ${ }^{40}$. Les scénarios produits médialement, comme ceux de la guerre du Golfe, dans lesquels on présenta aux téléspectateurs un jeu de lumière, ne doivent pas être confondus avec les villages potemkiniens qui, lorsqu'on y regarde de plus près et qu'on les examine plus précisément, se révèlent n'être que de la décoration. On ne peut soi-même ni regarder de plus près un phénomène téléprésent ni en considérer l'envers. Sur ce point, l'image télévisuelle ressemble à l'image représentative à laquelle Sartre reconnaît, à l'inverse du «foisonnement» des choses, une "pauvreté essentielle » puisque le lecteur ou celui qui regarde une oeuvre ne peut pas compléter un personnage de roman ou une veduta peinte grâce à d'autres expériences et ne peut pas les confirmer par des observations ${ }^{41}$.

intermédiaire entre l'intégration et la ségrégation, la complexité devient particulièrement grande » (M.-D. Weitze in Frankfurter Allgemeine Zeitung du 22.1. 1997, p. N3).

38. Hua, tome IV, p. $36 \mathrm{sq}$.

39. Théétète, $201 \mathrm{~b}$.

40. Au sujet de la distinction entre illusion perceptive et erreur perceptive, voir Max Scheler « Die Idole der Selbsterkenntnis », GW, Bd. 3, p. 222-226.

41. Sartre, 1940, p. 20. 
Au plan linguistique, à la perception correspond une proposition déictique du type : "C'est le Fujiyama ». Supposons qu'on mène quelqu'un, les yeux bandés, à la montagne. On lui enlève le bandage, on lui explique que «Là, c'est le Fujiyama » et on lui bande alors les yeux pour le chemin du retour. Il sait certes à quoi rassemble la montagne mais il ne sait toujours pas quelle est la montagne qui est le Fujiyama. D'autres montagnes pourraient lui ressembler; et ainsi, au sens strict, il ne peut pas retrouver ladite montagne ${ }^{42}$. Cela serait une illustration du fait que l'amputation rigoureuse des contextes débouche sur un quelque chose indéterminé spatialement, qui erre dans notre expérience comme un diablotin. D'une certaine manière, cette scène se répète plus ou moins lorsque nous recevons des informations des médias audiovisuels de l'image puisque nous sautons alors dans certains champs du présent comme dans un passé lointain. L'élargissement médial du champ de l'expérience se fait au prix d'un morcellement de l'expérience ${ }^{43}$.

La préparation sélective des scènes d'expérience se poursuit dans la technique de coupe qui intervient dans les relations d'expérience, dans le rythme de la succession des images, dans le choix de l'angle de vue ou du plan. Il faut toutefois admettre que toute expérience comporte une mise en perspective de la réalité. En percevant quelque chose en tant que quelque chose, de telle ou telle manière, nous ne prêtons pas attention à certains contextes. Ce n'est pas d'abord l'œil de la caméra qui est partiel, mais l'œil charnel. C'est pour cette raison que les médias artificiels sont, à l'inverse, susceptibles de nous apprendre quelque chose sur notre regard naturel. Plus inquiétant est un effet inverse qui apparaît lorsque les médias électroniques excèdent notre expérience charnelle par une démultiplication et une saturation des contextes. Un premier effet résiderait dans la suppression de la perspective par un regard total. Husserl se demande déjà s'il n'est pas possible que je voie tout de ma chair et qu'un œil voie l'autre œil ${ }^{44}$. Nous sommes proches de cette situation si - comme dans un «travail in situ » de Daniel Buren installé récemment dans la collection d'œuvres d'art de la Rhénanie-du-Nord-Westphalie - nous nous voyons en même temps dans plusieurs miroirs se réfléchissant et qu'ainsi nous nous regardons voir comme si nous étions une vidéo de nous-mêmes. Des philosophes peuvent se demander si cette chambre de miroirs ne peut pas être comprise comme la parodie de la conscience se réfléchissant elle-même. Il est pourtant décisif de se demander ce qui nous est ici donné à voir de nous-mêmes. Le trou optique, qu'Ernst Mach laisse en blanc de manière si efficace dans son autoportrait, se remplit-il ? Ne voyons-nous que ce qui est visible ou, audelà, voyons-nous le regard voyant? Avec cette question, nous nous

42. Sur cet exemple, voir Tugendhat et Wolf, 1993, p. 155 sq. Pour les auteurs, il s'agit là d'une utilisation identificatrice de termes singuliers.

43. Selon Husserl, les « morceaux » sont à comprendre comme des parties indépendantes (IIIle Recherche logique, § 1 sq.)

44. Hua, tome XIV, p. 518. 


\section{L'attention}

approchons d'une autre composante de l'expérience charnelle qui conduit au-delà des simples relations d'expérience.

\section{La délocalisation}

L'ici et maintenant charnel, à partir duquel je te regarde dans les yeux et te parle, se dessine comme une ride dans l'épais tissu de notre expérience. Husserl désigne la situation originaire du ici, qui ne représente pas une simple situation (situs) parmi d'autres, comme le «point zéro » de notre expérience $^{45}$ et, dans ses réflexions sur l'intersubjectivitét6, il qualifie à de multiples reprises le corps-chair propre de "corps zéro ». Les relations spatio-temporelles, avec leurs accents thématiques, seraient suspendues dans l'air comme le modèle du tapis volant si elles n'étaient pas centrées sur ce que Karl Bühler ${ }^{47}$ nomme l' « origo du champ déictique ». La possibilité à faire l'expérience de, qui est ce qui marque tout du sceau de la réalité, renvoie à ce lieu-temps dans lequel je séjourne charnellement et à partir duquel je me tourne vers ceci ou cela et me détourne du reste. Les différences de direction comme en haut/en-bas, devant/derrière et droite/gauche dépendent d'un tel centre d'orientation.

La localisation charnelle peut être atténuée et tendanciellement supprimée de deux manières. Une telle délocalisation se produit d'un côté par la dissolution de l'ici dans un présent dénué de contours et de limites, qui ne laisse qu'un sentiment diffus de l'ici et qu'un faible geste indicateur. Par là, le corps-chair se rapproche d'une chair purement sans corps, qui est entièrement auprès d'elle-même et qui n'a plus rien en dehors d'elle-même; et à cela correspond une réalité qui ne se détache plus de rien et qui, faute de déterminations différentielles, prend les traits d'une non-réalité. D'un autre côté, apparaît la possibilité d'un nivellement de l'ici à une position spatiale quelconque qui est encore connue mais qui n'est plus vécue comme adéquate. Le corps-chair se rapproche par là d'un simple corps sans chair. Lui correspond une réalité homogène qui perd également de son relief avec la mise à l'honneur de certains domaines et de certains aspects de la réalité. La personne qui perçoit se transforme en un "être de surfaces », comme le formule Husserl en relation avec Helmholtz ${ }^{48}$; et inversement, la réalité prend la forme d'une simple surface de projection. Au plan linguistique, à cette expérience limite bipolaire qui mène d'un côté à une chair sans corps et de l'autre à un corps sans chair, correspond une scission du discours qui met en évidence exclusivement le Je $(I)$ disant ou tout aussi exclusivement le Moi (me) dit.

La scission du Je et de la chair vient à notre rencontre de manière particulièrement impressionnante dans le domaine de la pathologie. Dans

45. Hua, tome IV, p. 158.

46. Cf. Hua, tome XIV.

47. Bühler, 1982, § 7 .

48. Hua, tome VI, p. 121. 
ses recherches sur le temps vécu ${ }^{49}$, Minkowski fait état d'une expérience avec deux patients à qui on soumet la question : "Où es-tu ? ». La double réponse qu'il obtient renvoie à une double perturbation. Un des deux patients, un paralytique, montre sans rien dire l'endroit où elle se tient alors que l'autre, un schizophrène, explique : «Je sais où je suis, mais je ne me sens pas y être ». Cette polarisation pathologique montre à nouveau certaines affinités avec des formes technologiques de la délocalisation. Les médias et leurs appareils descellent l'ancrage dans l'ici en libérant des possibilités de l'être-là-bas et en élargissant et en diversifiant l'espace de jeu de l'expérience. Un effet de déréalisation naît alors, par exemple lorsque le médium des images et des sons se transforme en un fluidum audiovisuel, en un flux d'images et de son dans lequel le spectateur et l'auditeur s'immergent. Cette expérience globale d'une immersion totale n'est pas dénuée de résonances religieuses et érotiques, à tout le moins auto-érotiques. D'un autre côté, il existe des tentatives pour saisir selon un modèle par niveaux la distance par rapport à la situation de la chair propre et la différence entre l'ici et le là-bas. Le vécu immédiat, que nous $n^{\prime}$ avons pas pour objet ${ }^{50}$, devient $l^{\prime}$ objet d'un métasavoir, d'un métalangage et d'une métavision qui se nomme observation. L'objectivation (Vergegenständlichung) suit une itération se poursuivant de manière quelconque dont les étapes peuvent être copiées sous la forme de stations relais. A l'instar de l'ici charnel, la "réalité » apparaît alors comme une catégorie d'observateur; ce qui se montre comme réel fait toujours partie d'un second ordre. Le savoir des choses (savoir de premier ordre) passe à un savoir au sujet des choses (savoir de deuxième ordre) et celui-ci débouche sur un savoir au sujet du savoir en tant que tel (savoir de troisième ordre). De là résulte une conséquence que Paul Watzlawick a formulée de la manière suivante : "Pour nous, la réalité est vraisemblablement ce qu'on considère comme réel ${ }^{51}$. »Cet énoncé n'est pas faux; mais ne s'y exprime pourtant qu'une demi-vérité. Le «tenir-pourréel » ne peut pas être réduit à un contenu d'observation ou de savoir d'un ordre supérieur et cela parce que l'auto-observation résulte d'un autoredoublement charnel ou - comme le constate Husserl du point de vue de la réflexion $-\mathrm{d}^{\prime}$ une scission et d'une démultiplication du moi ${ }^{52}$. Cela distingue la réflexion sensori-charnelle d'une simple récursion opérationnelle. Mais un effet de déréalisation n'apparaît pas seulement lorsque l'ici charnel - comme nous l'avons déjà mentionné - sombre dans un tout, mais également lorsqu'il est réduit à un là-bas qui peut être observé et enregistré. Selon Lucien Sfez ${ }^{53}$, dans le domaine des médias modernes de

49. Minkowski, 1968, p. 257.

50. Cf. Hua, tome III, p. 369 : « Nous ne voyons pas (...) ce que nous vivons actuellement. »

51. Cf. entre autres de Paul Watzlawick, 1969, p. 244. Le modèle communicationnel par strates est développé d'après le modèle russellien du saut des types, dans lequel les paradoxes sont finalement neutralisés au plan formel.

52. Hua, tome VII, p. 86-97.

53. Sfez, 1990, p. 412. 


\section{L'attention}

communication, il faut s'attendre à une "déréalisation réalisatrice ». La réalité se présente comme hyperréelle dans la mesure où elle est produite par des mécanismes de renvoi de niveau $\mathrm{x}$; et elle se présente en même temps comme hyporéelle dans la mesure où elle reconduit - loin de tout usage commun des signes - à une pure irruption de sens. Les deux formes de la délocalisation que nous avons distinguées se soutiennent ainsi mutuellement.

\section{La télé-expérience}

L'ici charnel ne fonctionne pas seulement comme "point zéro" de l'orientation spatiale mais aussi comme un "d'où » des automouvements spatio-temporels. Ce lieu de départ du mouvement représente un état de quiétude originaire ${ }^{54}$. En effet, la chair propre ne repose pas dans l'espace comme un corps qui ne se meut pas; un tel état de repos ne serait rien d'autre que la valeur limite négative d'un mouvement. La chair « repose » plutôt au sens où tous les mouvements qui parcourent l'espace ou qui se poursuivent en lui, sont mesurés à son aune ou sont ancrés en elle ${ }^{55}$. A l'égard des choses dans l'espace ou à d'autres êtres qui, quant à eux, sont « ici ", le mouvement charnel prend la forme d'un rapprochement ou d'un éloignement. Le proche et le lointain se mesurent à l'aune de la vitesse avec laquelle ces mouvements se font; et cette vitesse dépend quant à elle des techniques de déplacement. La technologie débouche sur une dromologie, comme Virilio la nomme; et une telle doctrine de la vitesse renvoie quant à elle à une phénoménologie du mouvement propre charnel. Le mouvement propre n'est en fin de compte pas un simple phénomène accessoire de la sensation et de la perception. Au contraire, celles-ci ne s'accomplissent ellesmêmes qu'en tant qu'un «je me meus», que Husserl désigne par le vieux terme de kinesthésie. Le «je sais » qui s'incarne dans la perception signifie toujours un «je peux ${ }^{56}$ ». Si on la conçoit comme la possibilité à faire l'expérience de, la réalité signifie concrètement que tout ce qui se manifeste comme réel est plus ou moins à notre portée. Un «monde à l'extérieur de notre monde ", qu'on ne pourrait pas atteindre par principe, se présenterait comme une simple possibilité logique ou reviendrait à un contresens logique ${ }^{57}$. Avec l'hypothèse d'un monde réel à l'extérieur de notre monde,

54. Hua tome XIV, p. 594.

55. Cela se transpose à la Terre en tant que sol sur lequel nous allons et nous nous tenons. Cf. Husserl (1940) : « Renversement de la doctrine copernicienne dans l'interprétation habituelle du monde. L'arche originaire Terre ne se meut pas ». Ou alors se meut-elle s'il nous arrive de « tomber vers le haut »? Cf. les sombres visions cosmiques de Virilio dans son œuvre, La vitesse de libération (1995).

56. Cf. Hua, tome IV, §60, Merleau-Ponty, 1945, p. 160. Voir en outre chez les auteurs anglosaxons la distinction courante entre knowing how et knowing that.

57. Hua, tome III, § 48 . 
nous nous réfèrerions en effet à une réalité dont on ne peut pas faire l'expérience et dont le caractère de réalité demeurerait une affirmation vide.

Avec Husserl, on peut néanmoins simuler une situation d'expérience dans laquelle le mouvement propre tend vers zéro ${ }^{58}$. Cette expérience de pensée rappelle la caverne platonicienne dans laquelle la réduction du monde à l'apparence optique va de pair avec le fait d'être rivé à un endroit. Husserl construit trois phases de l'expérience. La première phase est : «Une cellule. Ma chair sans le Je-peux de la locomotion kinesthésique. » La «cellule originaire» est selon Husserl 1' «espace orienté de la présence spatiale - qui précède la signification proche-lointain » (p. 539). Dans cette première phase, j'ai devant moi un simple espace proche qui est occupé par des choses proches. La deuxième phase : "Les murs tombent; je vois 'au lointain'. Le Je-peux de la locomotion manque à nouveau ». Nous n'accomplissons pas alors seulement une vision du proche (Nahsehen) mais aussi une télé-vision (Fernsehen ${ }^{59}$ ) avec des télé-choses (ou des choses télévisées, p. 540.) qui apparaissent dans un télé-espace, qui accomplissent des télé-mouvements et qui constituent une sorte de télé-réalité (Fernrealität). Les télé-choses ressemblent toutefois à des choses proches. Comme dans la représentation perspectiviste de la peinture, la lune prend dans le ciel la même surface qu'une pièce de monnaie dans ma main. Toutes les choses acquièrent le statut de ces «ultra-choses » qui sont pour l'enfant hors de portée non seulement du toucher mais aussi de la perception ${ }^{60}$. Mais en même temps, cela signifie que les choses ne perdent pas le statut de fantômes puisqu'elles sont issues de contextes de perception ayant fait leurs preuves, parmi lesquels les mises à l'épreuve actives par le mouvement propre. «C'est ainsi qu'il est certes possible de penser ce lointain comme réalité (...). Mais le lointain, en tant que réalité, n'est alors justement qu'une possibilité vide dont je ne peux pas dire qu'il est ou qu'il n'est pas » (p. 551). Plus précisément, on ne peut pas décider s'il s'agit de quelque chose de réel ou d'un simple fantôme. Ne s'y incarne ni une possibilité réelle ni une possibilité simplement fictive ou pensée, mais une indécidabilité dont on a fait l'expérience et qui est pour cette raison réelle - comme lorsque Descartes était enfermé dans son poêle et était ainsi réduit à un regard par la fenêtre. Chez Husserl, le charme disparaît dans une troisième phase dans laquelle je récupère la faculté de déplacement propre: "donc, je peux "aller" ", c'est-àdire «jusqu'au télé-horizon absolu du lointain visuel » (ibid.). Ce n'est que de cette façon que la réalité apparaît en tant que telle ; la question de savoir s'il s'agit de l'apparence ou de la réalité ne peut certes être tranchée dans tous les cas particuliers mais elle l'est bien de façon principielle.

58. Cf. Hua, tome XIV, p. 534-557 et spécialement pour ce qui suit, p. 550 sq.

59. Au sujet de la télé-perception et de la télé-vision en tant perception et vision au loin, voir Merleau-Ponty, 1960, p. $24 ; 1964$, p. 311, 327.

60. Ultra-chose est un concept d'Henri Wallon que Merleau-Ponty reprend dans ses conférences à la Sorbonne (1988, p. 242). 


\section{L'attention}

On peut alors se demander si ce qui est ici effectué en tant qu'expérience de pensée et qui vise à la préparation sélective des performances spécifiques de constitution ne reapparaît pas dans les arrangements techniques de l'expérience en tant $\mathrm{qu}^{\prime}$ «abstraction réelle». Le mouvement propre ne disparaît certes pas totalement ; mais il se réduit à une «mobilité sur place 61 » si une personne, assise dans un fauteuil devant l'écran, par la simple pression sur un bouton, "voit dans le lointain » ou « agit dans le lointain ». Il ne serait pas tout à fait faux de parler d'un réalisme magique lorsque la réalité obéit à une pression de la main et finalement aussi à un mot et qu'on se dispense du « contact » avec les choses ${ }^{62}$.

Un effet similaire à celui de l'absence relative de mouvement est alors atteint lorsque la vitesse du déplacement s'accroît en une hypervitesse et prend la valeur limite absolue de la vitesse de la lumière. Paul Virilio décèle le développement d'une proximité immédiate-charnelle qui, par le mouvement propre, conduit à une proximité mécanique rendue possible par l'usage de moyens de transport. Au-delà, elle débouche sur une proximité électromagnétique dans laquelle la différence entre ici et là-bas se dissout dans la simultanéité de l'ici et du là-bas et fait place à un « ailleurs communicationnel ${ }^{63}$ ». Avec la télé-présence qui se réalise sous la forme de télé-perception et de télé-action, le proche semble collaborer avec le lointain et le lointain avec le proche. De manière paradoxale, je suis moi-même là où je ne suis pas, et cela sous la forme d'une authenticité qui ne souffre aucun délai. Avec le retrait des horizons du monde apparaît pourtant un sentiment nouveau, celui d' «être cloîtré64 ». Nous serions cloîtrés dans la lumière sans ombre d'un présent qui pénètre tout. Cela correspond-il à une parodie tardive de la caverne de Platon?

Il existe cependant une ambigü̈té fondamentale dans le discours d'une téléprésence. La téléprésence peut signifier que même ce qui est loin est transféré dans la présence, donc que nos champs de perception et d'action s'étendent jusqu'au moindre recoin du monde et que des flux d'informations encercle le globe. Mais on oublie là l'absence qui est inscrite dans la présence. Le relief de l'expérience résulte du fait que les choses se trouvent en quelque sorte sur le chemin les unes des autres, qu'elles se disputent mon regard et luttent pour mon attention ${ }^{65}$. En tant que "télé-vision », la vision est une "cristallisation de $l^{\prime}$ impossible ${ }^{66} »$. Cela signifie que dès la perception naturelle ce qui est plus proche se dissocie de ce qui est plus loin; et cela vaut aussi pour la distance (Ferne) à moi-même qui résulte du fait que de toute ré-flexion se fait après coup. Cette modulation de l'expérience ne se modifie pas non plus si l'œil naturel s'arme d'un appareil photo ou d'une caméra vidéo. Le regard

61. Virilio, 1995.

62. Au sujet du destin de la main qui menace de déboucher sur une régression de la main, voir Leroi-Gourhan, 1984, p. 319 sq.

63. Virilio, 1995.

64. Ibid.

65. Merleau-Ponty, 1969, p. 74.

66. Merleau-Ponty, 1964, p. 327. 
dans le lointain se fait au détriment du regard jeté sur ce qui est proche. Pour les médias publics, cela signifie que les efforts pour la part d'audience se mêlent à une lutte continue pour l'attention des spectateurs et des auditeurs. Cette attention demeure également limitée si les boîtes servant à ranger les vidéos se remplissent. On peut zapper entre les programmes télévisés comme on feuillette un livre; mais lire et voir ne commencent vraiment que lorsque le regard s'accroche quelque part. La démultiplication des points de vue de l'expérience et le relâchement du lien à l'ici charnel ne supprime pas la contingence des points de vue. Au contraire, le problème de la sélection croît fortement avec les possibilités techniques. Cela ne peut amener qu'à désavouer celui qui confond l'enregistrement de données avec leur traitement dans l'expérience ou l'augmentation des capacités d'enregistrement avec le souvenir, l'effacement de données avec l'oubli ${ }^{67}$. Si on fait cette confusion, la réalité de l'expérience se réduit à des mécanismes correspondants de représentation, de production et de transfert de telle sorte que le fonctionnement de la machine peut finalement passer pour une preuve de la réalité. Pour ces appareils vaudrait ce que Platon disait au sujet des philosophes : "son corps seul est présent et séjourne dans la ville, tandis que sa pensée $(. .$.$) promène partout son \mathrm{vol}^{68} »$. A notre époque, cela voudrait dire que seul le matériel informatique stocke en un certain endroit tandis que le logiciel bruisse dans tout le réseau des ordinateurs à la recherche de connexions. Le seul reste d'étrangeté (Fremdheit) ne serait alors que le non-lieu des techniques téléoptiques (interface homme/machine, centre de contrôle ou point nodal du transfert de données, etc ${ }^{69}$.) ».

\section{La virtualisation}

Comme la réalité, la possibilité doit aussi être définie comme une modalité impliquée de l'expérience et non comme l'attribut d'une chose dont on peut faire l'expérience ou qu'on peut nommer. Dans le domaine de l'expérience, la virtualisation signifie qu'on fait l'expérience de, $q u^{\prime}$ on considère ou manie quelque chose comme possible. Si nous considérons que la réalité dont nous pouvons faire l'expérience offre toujours des espaces de jeu pour la possibilité, c'est-à-dire qu'elle ne s'accomplit jamais comme une pure et pleine réalité, la potentialité ou la virtualité ${ }^{70}$ ne contrastent pas d'abord avec la réalité mais avec l'actualité. Il nous faut ici distinguer les possibilités au sein d'une situation concrète et les situations possibles. Il en va de même pour les structures et les règles ouvertes qui, d'une part, ouvrent et excluent des possibilités et qui, d'autre part, apparaissent elles-mêmes comme des structures ou des règles possibles parmi d'autres.

67. À ce sujet, voir Käte Meyer-Drawe, 1996, : « Die Untreue des Gedächtnisses »

68. Théétète 173 e.

69. Virilio, 1995

70. Il s'agit d'ailleurs d'un terme très masculin : vir-tus = force de l'homme. 


\section{L'attention}

Les découvertes pathologiques faites par Kurt Goldstein, Adhémar Gelb et leurs collaborateurs avec le cas Schneider montrent quelle signification il faut accorder à cette distinction. Ce patient, qui avait été blessé par un éclat d'obus dans la zone optique du cerveau, se montre très limité dans ses possibilités de comportement. Il est certes encore capable de faire face, dans l' "attitude concrète», aux exigences immédiates de la situation; mais il a perdu la capacité «de bien se rapporter à ce qui n'est que possible» et $\mathrm{d}^{\prime}$ adopter une attitude «abstraite» ou «catégoriale ${ }^{71}$ ». Il peut ainsi s'orienter de manière quotidienne dans l'espace et dans le temps sans qu'il ne soit en mesure d'indiquer la distance ou des données temporelles. Il n'a aucune difficulté à chasser une mouche qui le pique; mais il échoue lorsqu'on lui demande de montrer avec le doigt une partie de son corps et pas seulement de saisir avec une certaine position du corps. Il est en mesure de raconter ce qu'il a lui-même vécu; mais il ne parvient pas à restituer des vécus étrangers et à décrire ou présenter des scènes possibles. Il manque d'initiative dans les relations sexuelles comme dans la conversation ; le jeu et les blagues laissent place à un sérieux visible qui s'en tient à ce qui est nécessaire et à ce qui s'impose. La « perte de la catégorie de la possibilité » se révèle être une «perte de la liberté ${ }^{72}$ ». Selon Merleau-Ponty, qui analyse minutieusement le cas, le malade reste «enfermé dans l'actuel $^{73}$ » alors que chez les personnes normales la chair fonctionne comme «centre d'action virtuel ${ }^{74}$ » et qu'un «espace virtuel ou humain » se superpose à l'espace physique ${ }^{75}$. Avec Robert Musil, on peut considérer le penchant du patient à l'attitude concrète comme l'expression d'un sens exagéré de la réalité né d'un besoin d'orientation. Si nous désignons un tel comportement comme le « sérieux animal », nous serons sûrement injuste envers le comportement de jeu propre aux animaux; mais cela pourrait nous rendre attentif aux traits comparables dans le comportement des animaux semblables aux hommes. Wolfgang Köhler attribue aux chimpanzés, qui utilisent par exemple des bâtons pour faire descendre des bananes, un comportement qui convient à l'immédiat mais qui n'est pas adapté au virtuel ${ }^{76}$.

Les champs actuels d'expérience présentent des espaces de jeu ouverts qui peuvent être employés diversement. Ils comportent des zones grises et vides d'indétermination qui ne peuvent cependant jamais être comblées de telle ou telle façon ${ }^{77}$. Enfin, en raison de leur constitution contingente, les champs d'expérience offrent des possibilités de réorganisation et de

71. Goldstein, 1934, p. 18 sq. À ce sujet, Merleau-Ponty, 1949, p. 69.

72. Goldstein 1934, p. 198.

73. Merleau-Ponty, 1945, p. 126.

74. Ibid., p. 127.

75. Ibid., p. 129

76. Cf. Wolfgang Köhler, Intelligenzprüfungen am Menschenaffen. À ce sujet, voir Merleau-Ponty, 1949, p. 122-130, p. 189 sq.

77. Sur la conception moderne d'une indétermination positive, voir Gerhard Gamm, Flucht aus der Kategorie (1994). 
restructuration. «Ce qui définit l'homme n'est pas la capacité de créer une seconde nature - économique, sociale, culturelle - au-delà de la nature biologique, c'est plutôt celle de dépasser les structures créées pour en créer $\mathrm{d}^{\prime}$ autres ${ }^{78}$ ». Le chemin vers la virtualisation ne s'ouvre que si nous faisons un pas de plus et ne prenons pas seulement en compte des restructurations possibles de ce monde mais activons des mondes et des espaces possibles dans lesquels nous basculons comme dans un autre monde. Ce basculement ne signifie lui aussi rien de complètement nouveau tant que le monde possible est compris comme un monde imaginaire, comme un monde de la fiction qui demeure rattaché au monde réel sur le mode du comme si. Qui entre dans le «monde des Guermantes » n'acquiert aucun titre de noblesse reconnu par sa propre société ; et le lecteur de Scènes de vie d'un propre à rien ne peut compter sur aucun bonus littéraire auprès du bureau des déclarations et des services fiscaux. La question de savoir comment, dans de tels cas, la réalité et la fiction s'entrelacent est une question importante dont nous pouvons faire abstraction dans ce contexte. Ce qui nous intéresse ici est le problème déjà mentionné d'une réalisation technique de fictions. L'expérience d'un cyberespace produit électroniquement peut être interprétée à partir de l'expérience sensorielle comme un processus limite dans lequel la similitude croît rapidement ${ }^{79}$. L'écran bidimensionnel, devant lequel nous nous trouvons, ou l'espace-image, qui s'ouvre devant nous, se conjuguent ultimement en un espace tridimensionnel autour de nous dans lequel nous pouvons regarder à l'aide de casques digitaux, agir à l'aide de gants digitaux et de robots pilotés, dans lequel nous nous saluons avec le "feedback-glove» et peut-être essayons une "masturbation médiale » à deux ${ }^{80}$. Il ne s'agit pas ici d'un élargissement du monde marquant (Merkwelt) et du monde de l'agir-sur (Wirkwelt) mais d'un environnement crée artificiellement dans lequel remarquer (bemerken) et la produire (bewirken) sont couplés. L'expérience du cyberespace se transformerait totalement en une cyberexpérience non seulement si, comme dans le cas du projet Cyborg, certains organes naturels étaient remplacés par des organes plus forts ou plus raffinés, mais si le corps dans sa totalité était transféré dans une machine corporelle fonctionnant électroniquement ${ }^{81}$. Là aussi le soi charnel devrait entrer dans le cyberespace comme le moine méditant qui est englouti par la montagne ; et le cyberartiste devrait réussir non seulement à produire un Pygmalion mais aussi à se transformer lui-même en un Pygmalion.

78. Merleau-Ponty, 1949, p. 189. Au sujet de l'organisation et de la réorganisation des champs d'expérience, qui dépassent systématiquement le cadre d'une phénoménologie de l'acte noétique, voir Gurwitsch, Das Bewusstseinsfeld (1975).

79. Il faut néanmoins indiquer que la création de ressemblance (Verähnlichung) ne constitue que l'une des fonctions de la mise en image ; l'autre réside dans l'objectivation, dans l'évocation des dieux absents, des souverains, des morts ou des personnes aimées.

80. Virilio, 1995

81. Derrière l'échangeabilité du corps se cache le « rêve cybernétique d'un esprit sans corps » (Meyer-Drawe, 1996, p. 191). 


\section{L'attention}

De sobres techniciens, qui aménagent le cyberespace pour des simulations de vol ou des opérations chirurgicales à distance et créent ainsi des enclaves virtuelles, rejetteront de telles représentations comme étant de la science-fiction. L'espace, dans lequel est installé le cyberespace, est tout aussi peu un cyberespace que le lit dans lequel s'accomplit la fin d'un rêve est un lit rêvé. On peut masquer le cadre dans lequel se déroule le virtuel ; mais on ne peut pas le supprimer. Enfin, la nourriture artificielle ou la fécondation artificielle ne sont pas de la nourriture ou de la fécondation virtuelles. $\mathrm{Si}$, dans un phénomène par contraste comme veiller/dormir ou actualité/virtualité, on supprime l'un des membres, le membre opposé disparaît également. Les visions pantechniques rencontrent ici leurs limites. Pourtant, ces limites sont toujours à penser à nouveaux frais car les visions ne sont efficaces que parce qu'elles sont des visions. Husserl, qui accorde une si grande importance à notre expérience actuelle, considère toutefois, dans ses recherches eidétiques, la fiction comme l'« élément vivant de la phénoménologie ${ }^{82} »$. Dans les Méditations cartésiennes $(\S 37,41)$, il distingue la variation liée qui, dans la simulation de l'expérience, fixe à un monde factuel, à un Je factuel et à Nous factuel, de la variation sans lien qui renonce à tout lien avec les faits en considérant le monde réel - qui comprend le Je et le Nous factuels - comme une pure possibilité. Pourtant, de façon significative, Husserl n'en est pas resté à cette manière de voir inspirée par Leibniz. Dans ses notes du Nachlass, il se fait à lui-même l'objection que dans le cas du monde et du Je, la réalité de la possibilité prévaut, en tant que fait «absolu " incontournable, qui est nécessaire pour prêter aux possibilités imaginaires la qualité des possibilités réelles ${ }^{83}$.

On pourrait prolonger les pensées de Husserl en disant que des " virtualités pures » seraient des virtualités simplement pensées. Mais cela n'exclut pas que l'importance ne soit déplacée que du sens de la réalité vers le sens de la possibilité. De la même manière que « l'attitude concrète » visà-vis de ce qui est proche et normal peut se figer en une "attitude concrétiste ", l'attitude "abstraite» peut se muer en attitude fictionnelle 84 . La domination de ces catégories et de ces symboles que Goldstein a en vue serait remplacée par celle de constructions, de codes, de médias et d'appareils technologiques, qui n'admettent le réel que dans la mesure où il peut être représenté et transmis médialement, mais aussi manipulé digitalement sous cette forme réduite. Il faut toutefois faire observer que ce n'est seulement dans les cas exceptionnels que les processus technologiques échappent à notre contrôle ; cela se produit à chaque fois qu'ils quittent le champ délimité des systèmes clos des laboratoires. Un «morceau de nature » revient toujours par la porte de derrière. Le libre arbitre, auquel

82. Hua, tome III, p. 163.

83. Cf. Hua, tome XV, p. 519.

84. Le primat accordé par Goldstein à l'«attitude catégoriale » vis-à-vis $l^{\prime}$ «attitude concrète » est critiqué par Merleau-Ponty comme un préjugé intellectualiste (1945, p. 140-162). À ce sujet, au plan neuropsychologique, voir les objections de Lurija, 1992, p. 334 sq. 
Husserl a recours pour ses variations imaginatives, demeure trop orienté vers le faire. Les processus qui ne sont pas simplement «à l'œuvre » mais qui sont mis "en scène » ne peuvent être pilotés que de façon limitée. Cela vaut déjà pour les actions les plus simples qui sont guidées par des perceptions sensorielles et qui sont portées par des mouvements charnels. Là où quelque chose est mis en scène, il y a des joueurs, des coéquipiers et des adversaires. Le metteur en scène n'est pas un producteur; il n'est qu'un protagoniste $^{85}$.

D'autres réflexions pourraient montrer que, dans les expérimentations avec la réalité, nous ne nous heurtons pas seulement aux limites de ce que l'ordinateur et nous, en tant que coéquipiers ou adversaires, ne pouvons pas (encore) faire. Nous nous exposons à des perturbations et à de possibles catastrophes qui entravent nos opérations et transgressent nos règles. Enfin, s'annoncent des exigences étrangères qui nous échappent du fait qu'elles suscitent des réponses possibles. La question bien connue de savoir de quoi sont capables les ordinateurs et de quoi ils ne sont pas capables, culminerait dans la question: les ordinateurs peuvent-ils répondre, c'est-à-dire pas simplement réagir mais s'occuper de l'étranger? Le ne-pas-pouvoir-faire, qui est inscrit dans le pouvoir-faire propre, signifierait que le faire propre commence ailleurs. Il y a des expérimentations qui nous adviennent avant que nous procédions nous-mêmes à des expérimentations. ${ }^{86}$

Traduit par Flavien Le Bouter

\section{Bibliographie}

Bühler, K., Sprachtheorie, Stuttgart/New York, Fischer, 1982

Foerster, H. von, Wissen und Gewissen, Francfort/Main, Suhrkamp, 1993

Gamm, G., Flucht aus der Kategorie. Die Positivierung der Unbestimmheit als Ausgang der Moderne, Francfort/Main, Suhrkamp, 1994

Glasersfeld, E. von, Radikaler Konstruktivismus, Francfort/Main, Suhrkamp, 1996

Goldstein, K., Der Aufbau des Organismus, La Haye, Nijhoff, 1934 (trad. fr. La structure de l'organisme, Paris, Gallimard, 1951)

Gurwitsch, A., Das Bewusstseinsfeld, Berlin/New York, De Gruyter, 1975

85. Au sujet du concept de "mise en scène », en tant que jonction entre l'action et la passion, voir Hua, tome IV, p. 98, 259, 336. F.J. Varela et E. Thompson (1992, chap. 8) reprennent cette pensée de Merleau-Ponty et en font un usage fécond pour une conception des cognitions incarnées.

86. Note des éditeurs : le texte de Bernhard Waldenfels, Topographie de l'étranger. Essais sur une phénoménologie de l'étranger, traduit par F. Gregorio, F. Moinat, A. Renken, M. Vanni est paru en 2009 aux éditions Van Dieren, Paris. 


\section{L'attention}

Husserl, E., «Grundlegende Untersuchungen zum phänomenologischen Ursprung der Räumlichkeit der Natur» in Philosophical Essays in Memory of E. Husserl, M. Farber (éd.), Cambridge, Mass, Harvard University Press, 1940

Husserl, E., Husserliana, La Haye/Dordrecht, Nijhoff (= Hua)

James, W., The Principles of Psychology, 2 tomes, New York, Dover Publ., 1950

James, W., Essays in Radical Empirism in The Works of William James, tome 3, Cambridge, Mass./Londres, Harvard University Press, 1976

Köhler, W., Intelligenzprüfungen an Menschenaffen, Berlin/Göttingen/Heidelberg, 1921 (trad. fr. L'intelligence des singes supérieurs, Paris, Alcan, 1927)

Leroi-Gourhan, A., Le geste et la parole, Paris, Albin Michel, 1964-1965

Lurija, A.R., Das Gehirn in Aktion, Reinbek, Rowohlt, 1992

Merleau-Ponty, M., Phénoménologie de la perception, Paris, Gallimard, 1945

Merleau-Ponty, M., La structure du comportement, Paris, PUF, 1949

Merleau-Ponty, M., Le visible et l'invisible, Paris, Gallimard, 1964

Merleau-Ponty, M., La prose du monde, Paris, Gallimard, 1969

Merleau-Ponty, M., Merleau-Ponty à la Sorbonne. Résumés de cours 1949-1952, Cynara, 1988

Meyer-Drawe, K., Menschen im Spiegel ihrer Maschinen, Munich, Fink, 1996

Minkowski, E., Le temps vécu, Neuchatel, éd. Delachaux \& Niestlé, 1968

Musil, R., Der Mann ohne Eigenschaften, Reinbek, Rowohlt, 1978

Rickert, H., Die Grenzen der naturwissenschaftlichen Begriffsbildung, Tübingen, Mohr/Siebeck, 1913

Ros, A.I. «'Wirklichkeit' und 'Konstruktion'. Der Status der Wirklichkeit in der Genese kognitiver Strukturen bei Jean Piaget. II. 'Konstruktion' und 'Wirklichkeit'. Bemerkungen zu den erkenntnistheoretischen Grundannahmen des Radikalen Konstruktivismus » in G. Rusch et S.J. Schmidt (éd.), Piaget und der radikale Konstruktivismus, Francfort/Main, Suhrkamp, 1994

Sartre, J.P., L'imaginaire, Paris, Gallimard, 1940

Scheler, M., «Die Idole der Selbsterkenntnis » in Gesammelte Werke, Bd. 3, Vom Umsturz der Werte, Berne/Munich, Francke, 1972

Sfez, L., Critique de la communication, Paris, Seuil, 1990

Tugendhat, E., Vorlesungen zur Einführung in die sprachanalytische Philosophie, Francfort/Main, Suhrkamp, 1976

Tugendhat, E., Wolf, U., Logisch-semantische Propädeutik, Stuttgart, Reclam, 1993

Varela, J.V., Thompson, E., Der mittlere Weg der Erkenntnis, Berne/Munich, Vienne, Scherz, 1992 


\section{Expérimentations avec la réalité}

Virilio, P., La vitesse de libération, Paris, Galilée, 1995

Waldenfels, B., Phänomenologie in Frankreich, Francfort/Main, Suhrkamp, 1983

Waldenfels, B., In den Netzen der Lebenswelt, Francfort/Main, Suhrkamp, 1985

Waldenfels, B., Antwortregister, Francfort/Main, Suhrkamp, 1994

Waldenfels, B., Grenzen der Normalisierung. Studien zur Phänomenologie des Fremden, tome 2, Francfort/Main, Suhrkamp, 1998, édition augmentée 2008

Watzlawick, P., Beavin, J.H., Jackson D.D., Une logique de la communication, Paris, Seuil, 1972 